\title{
Controller design for global fixed-time synchronization of delayed neural networks with discontinuous activations
}

\author{
Leimin Wang ${ }^{\mathrm{a}}$, Zhigang Zeng ${ }^{\mathrm{b}, *}$, Junhao $\mathrm{Hu}^{\mathrm{c}}$, Xiaoping Wang ${ }^{\mathrm{b}}$ \\ ${ }^{a}$ School of Automation, China University of Geosciences, Wuhan 430074, China \\ ${ }^{b}$ School of Automation, Huazhong University of Science and Technology, Wuhan 430074, China \\ ${ }^{c}$ College of Mathematics and Statistics, South-Central University for Nationalities, Wuhan 430074, China
}

\begin{abstract}
This paper addresses the controller design problem for global fixed-time synchronization of delayed neural networks (DNNs) with discontinuous activations. To solve this problem, adaptive control and state feedback control laws are designed. Then based on the two controllers and two lemmas, the error system is proved to be globally asymptotically stable and even fixed-time stable. Moreover, some sufficient and easy checked conditions are derived to guarantee the global synchronization of drive and response systems in fixed time. It is noted that the settling time functional for fixed-time synchronization is independently on initial conditions. Our fixed-time synchronization results contain the finite-time results as the special cases by choosing different values of the two controllers. Finally, theoretical results are supported by numerical simulations.
\end{abstract}

Keywords: Delayed neural networks, discontinuous activations, global fixed-time synchronization, adaptive control, state feedback control

\section{Introduction}

Synchronization is a foundation to cognize an unknown dynamical system via another well-known dynamical system since it generally indicates the state trajectories of two systems are in accord with each other as time varies. Recently, synchronization of DNNs has been a hot topic for its potential applications in secure communication and signal processing. Lots of asymptotic and exponential synchronization results have been obtained under varied control approaches, such as linear state feedback control (Wang et al., 2013b; Wen \& Zeng, 2013; Wu \& Zeng, 2012; Zhang et al., 2013), adaptive control (Wang et al., 2015; Zhang et al., 2007), delay control (Karimi \& Gao, 2010; Wang \& Shen, 2015b), sample data control (Wu et al., 2012) and intermittent control (Zhang et al., 2015).

As is well known, the neuron activation function plays an important role in the dynamical analysis of DNNs. As the extension of continuous activations, discontinuous activations can be better applied to the DNNs model when the gain of the neuron amplifiers is very high (Forti \& Nistri, 2003). Moreover, DNNs with discontinuous activations possess potential applications in impacting machines, power circuits and optimization problem (Chandrasekar et al., 2015; Forti \& Nistri, 2003; Forti et al., 2005; Guo \& Huang, 2009; Liu et al., 2011, 2014; Wang et al., 2009; Yang et al., 2015). Therefore, it is of great significance to consider the discontinuous activations in DNNs.

\footnotetext{
${ }^{*}$ Corresponding author. Tel.: +86 87543630 .

Email addresses: leiminw89@126.com (Leimin Wang), zgzeng@hust.edu.cn (Zhigang Zeng), junhaohu74@163.com (Junhao Hu), wangxiaoping@hust.edu.cn (Xiaoping Wang)

Preprint submitted to Elsevier

Finite-time convergence has attracted increasing attentions since it satisfies the time response in some reality circumstances (Bhat \& Bernstein, 2000; Efimov et al., 2014; Moulay et al., 2008). Finite-time convergence offers the state convergence with finite settling time to the equilibrium state, thus it is better than the asymptotical or exponential convergence with infinite settling time. For the finite-time stability or synchronization of neural networks, lots of criteria have been established by using different control methods (Abdurahman et al., 2015; Hu et al., 2014; Huang et al., 2014; Jiang et al., 2015; Liu et al., 2014; Mei et al., 2013; Shen \& Cao, 2012; Velmurugan et al., 2016; Wang et al., 2016; Wang \& Shen, 2015a; Yang et al., 2015). Based on the delayed feedback control, the finite-time convergence of DNNs was studied with continuous activations in Abdurahman et al. (2015); Hu et al. (2014) and with discontinuous activations in Wang et al. (2016), respectively. Under discontinuous activations and controller, Liu et al. (2014) addressed the finite-time stabilization problem of neural networks via nonsmooth analysis. Mei et al. (2013) designed an effective feedback control with an updated law to actualize the synchronization in finite time between two chaotic neural networks. Under the nonlinear controller, the finite-time stabilizability problem was discussed for memristive DNNs in Wang \& Shen (2015a).

The settling time for finite-time convergence heavily depends on the initial conditions, which gives rise to different convergence times under different initial conditions. As the extension of finite-time convergence, the fixed-time convergence implies the settling time is bounded by some positive constant (Polyakov, 2012; Polyakov et al., 2015). In other words, the settling time is independent of initial conditions and then is not under the influence of initial conditions. The fixed-time 
synchronization of delayed Cohen-Grossberg neural networks was studied in Wan et al. (2016). However, the activation functions in that paper are required to be Lipschitz continuous and bounded. To the best of authors' knowledge, so far, few precise results have been reported for the fixed-time convergence of DNNs with discontinuous activations. The reasons or difficulties are listed in two aspects. On the one hand, the DNNs model with discontinuous activations is a delayed system with discontinuous right-hand side, so it is not easy to design proper controllers that can realize the synchronization of drive-response systems in fixed time. On the other hand, fixed-time convergence is difficult to deal with and it is hard to find a Lyapunov functional which fulfils the strict condition compared with the exponential convergence. Although some control laws such as linear control and adaptive control are successfully adopted in studying exponential synchronization of DNNs with or without discontinuous activations, they are not easy applied to handle the finite-time or fixed-time synchronization of DNNs.

This paper focus on the controller design problem for global fixed-time synchronization of DNNs with discontinuous activations. In order to realize the synchronization of the driveresponse systems in fixed time, two control laws include the adaptive control and state feedback control are designed. The main contributions of this paper are summarized as follows. Firstly, time delays and discontinuous activations are considered in studying the fixed-time synchronization of neural networks. Secondly, the settling time of fixed-time synchronization is independently on initial conditions, which makes it flexible for the adjustment of system control in industry. Thirdly, adaptive controller can adjust coupling strength automatically and it gets effective results in practical applications (Mei et al., 2013; Wang et al., 2015; Yang et al., 2015). So a novel adaptive control law is designed to study the fixed-time synchronization of DNNs with discontinuous activations. Fourthly, global fixedtime synchronization of DNNs with discontinuous activations is realized via the designed adaptive control and state feedback control methods. The two control methods can be used to study other nonlinear delayed systems with or without discontinuous right-hand sides.

The rest of this paper is organized as follows. Some preliminaries are introduced in Section 2. In Section 3, we designed the adaptive control and state feedback control laws. Then global fixed-time synchronization of DNNs with discontinuous activations is realized by using the two control laws. In Section 4, two examples are provided to demonstrate the effectiveness of the obtained results. Finally, Section 5 shows the conclusion.

Notations: $R_{+}, R^{n}$ and $R^{n \times n}$ denote the set of all nonnegative real numbers, the $n$-dimensional Euclidean space and the set of all $n \times n$ real matrices, respetively. For a given square matrix $A=\left(a_{i j}\right)_{n \times n} \in R^{n \times n},|A|=\left(\left|a_{i j}\right|\right)_{n \times n}$. For a function $f: R \rightarrow R$, if the right and left limits $f^{+}(x), f^{-}(x)$ of its point $x$ exist, $K[f(x)]=\left[\min \left\{f^{-}(x), f^{+}(x)\right\}, \max \left\{f^{-}(x), f^{+}(x)\right\}\right]$. A continuous function $\digamma: R \rightarrow R$ belongs to the class $\mathbb{K}$ if it is strictly increasing and $\digamma(0)=0$. And it belongs to the class $\mathbb{K}_{\infty}$ if it is strictly increasing, radially unbounded and $\digamma(0)=0$. $\operatorname{diag}\{\cdots\}$ denotes a block-diagonal matrix. $\operatorname{sign}(\cdot)$ is the classical signum function.

\section{System description and preliminaries}

In this paper, we consider a class of DNNs as follows:

$$
\dot{x}(t)=-D x(t)+A f(x(t))+B f(x(t-\tau(t)))+I,
$$

where $x(t)=\left(x_{1}(t), x_{2}(t), \ldots, x_{n}(t)\right)^{T} \in R^{n}$ and $f(x(\cdot))=$ $\left(f_{1}\left(x_{1}(\cdot)\right), f_{2}\left(x_{2}(\cdot)\right), \ldots, f_{n}\left(x_{n}(\cdot)\right)\right)^{T} \in R^{n}$ denote the neuron state and the activation function, respectively. $D=$ $\operatorname{diag}\left(d_{1}, d_{2}, \ldots, d_{n}\right)$ is a diagonal matrix with $d_{i}>0(i=$ $1,2, \ldots, n) . A=\left(a_{i j}\right)_{n \times n}, B=\left(b_{i j}\right)_{n \times n} \in R^{n \times n}$ are the connection weight matrix and delayed connection weight matrix, respectively. $I=\left(I_{1}, I_{2}, \ldots, I_{n}\right)^{T}$ is the vector of constant neuron inputs. $\tau(t)$ is the time-varying delay satisfying $0 \leq \tau(t) \leq \tau, \tau$ is a constant.

The following assumptions are given for DNNs (1).

(A1) For every $j=1,2, \ldots, n, f_{j}: R \rightarrow R$ is continuous except on a countable set of isolate points $\left\{\rho_{k}^{j}\right\}$, where the finite right and left limits $f_{j}^{+}\left(\rho_{k}^{j}\right)$ and $f_{j}^{-}\left(\rho_{k}^{j}\right)$ exist.

(A2) For each $j=1,2, \ldots, n$, there exist constants $h_{j}>$ $0, r_{j}>0$ such that

$$
\sup \left|\Psi_{j}-\chi_{j}\right| \leq h_{j}|u-v|+r_{j},
$$

for all $u, v \in R$, where $\Psi_{j} \in K\left[f_{j}(u)\right], \chi_{j} \in K\left[f_{j}(v)\right]$.

Remark 1. As the extension of continuous activations, discontinuous activations can be better applied to the DNNs model when the gain of the neuron amplifiers is very high (Forti \& Nistri, 2003). The authors in Forti \& Nistri (2003) introduced four kinds of discontinuous activations, including hard comparator (signum) function, piecewise linear monotonically nondecreasing function, discontinuous strictly increasing function and multilevel function.

Since DNNs (1) is with discontinuous right-hand side, we need to specify what is the solution of (1).

Definition 1 (Forti et al., 2005). A function $x:[-\tau, T) \rightarrow$ $R^{n}, T \in(0,+\infty]$, is a solution (in Filippov's sense) of DNNs (1) on $[-\tau, T)$, if:

i) $x$ is continuous on $[-\tau, T)$ and absolutely continuous on $[0, T)$;

ii) there exists a measurable function $\chi=\left(\chi_{1}, \chi_{2}, \ldots, \chi_{n}\right)^{T}$ : $[-\tau, T) \rightarrow R^{n}$, such that

$$
\begin{aligned}
& \dot{x}(t)=-D x(t)+A \chi(t)+B \chi(t-\tau(t))+I, \\
& \text { and } \quad \chi(t) \in K[f(x(t))], \quad \text { for a.a. } t \in[0, T) .
\end{aligned}
$$

Definition 2 (Forti et al., 2005). For any continuous function $\varphi:[-\tau, 0] \rightarrow R^{n}$ and any measurable selection $\iota(s) \in$ $K[f(\varphi(s))]$ for a.a. $s \in[-\tau, 0]$ associated to (1) with initial condition $(\varphi, \iota)$, the initial value problem means finding a couple of functions $[x, \chi]:[-\tau, T) \rightarrow R^{n} \times R^{n}$, such that $x$ is a solution of (1) on $[-\tau, T)$ for some $T>0, \chi$ is the output associated to $x$, and

$$
\left\{\begin{array}{rlrl}
\dot{x}(t)= & -D x(t)+A \chi(t)+B \chi(t-\tau(t))+I, \\
& \text { for a.a. } t \in[0, T) \\
\chi(t) \in & K[f(x(t))], \quad & \text { for a.a. } t \in[0, T) \\
x(s)= & \varphi(s), & \quad \forall s \in[-\tau, 0], \\
\chi(s)= & \iota(s), & \text { for a.a. } s \in[-\tau, 0] .
\end{array}\right.
$$


Consider DNNs (1) as the drive system. Then the corresponding response system is

$$
\dot{z}(t)=-D z(t)+A f(z(t))+B f(z(t-\tau(t)))+I+u(t),
$$

where $z(t)=\left(z_{1}(t), z_{2}(t), \ldots, z_{n}(t)\right)^{T} \in R^{n}$ is the state variable of DNNs $(4), u(t)=\left(u_{1}(t), u_{2}(t), \ldots, u_{n}(t)\right)^{T}$ is the controller to be designed, the other parameters are the same as in (1).

Definition 3 (Forti et al., 2005). For any continuous function $\psi:[-\tau, 0] \rightarrow R^{n}$ and any measurable selection $\omega(s) \in$ $K[f(\psi(s))]$ for a.a. $s \in[-\tau, 0]$ associated to (4) with initial condition $(\psi, \omega)$, the initial value problem means finding a couple of functions $[z, \Psi]:[-\tau, T) \rightarrow R^{n} \times R^{n}$, such that $z$ is a solution of (5) on $[-\tau, T)$ for some $T>0, \Psi$ is the output associated to $z$, and

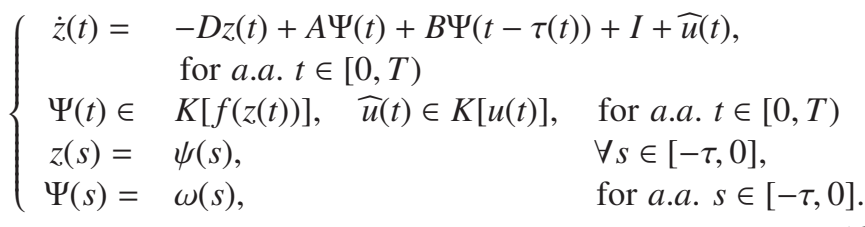

Define the synchronization error as $e(t)=$ $\left(e_{1}(t), e_{2}(t), \ldots, e_{n}(t)\right)^{T}, e_{i}(t)=z_{i}(t)-x_{i}(t), i=1,2, \ldots, n$. Then from (4) and (6), we can obtain the following synchronization error system:

$$
\dot{e}(t)=-D e(t)+A \zeta(t)+B \zeta(t-\tau(t))+u(t),
$$

where $\zeta(t)=\left(\zeta_{1}(t), \zeta_{2}(t), \ldots, \zeta_{n}(t)\right)^{T}=\Psi(t)-\chi(t), u(t)=$ $\left(u_{1}(t), u_{2}(t), \ldots, u_{n}(t)\right)^{T}$ is the control input that will be designed latter.

In order to obtain the sufficient condition for the finite-time synchronization of DNNs (1) and (5), the following definitions and useful lemmas are provided.

Definition 4 (Moulay et al., 2008). The error system (7) is said to be globally stable in finite time if for any initial condition $\phi(s)=\psi(s)-\varphi(s) \in R^{n}$, there exists $0 \leq T(\phi)<+\infty$ such that $e(t, \phi)=0$ for all $t \geq T(\phi) . T_{0}(\phi)=\inf \{T(\phi) \geq 0: e(t, \phi)=$ $0 \quad \forall t \geq T(\phi)\}$ is a functional called the settling time of the (7).

Definition 5 (Polyakov, 2012). DNNs (1) and (5) are said to be globally synchronized in fixed time if the error system (7) is global finite-time stable and the settling time functional $T_{0}(\phi)$ is global bounded by some positive constant $T_{\max }>0$, i.e. $T_{0}(\phi) \leq T_{\max }$ for $\phi \in R^{n}$.

Lemma 1 (Hardy et al., 1988). If $x_{1}, x_{2}, \ldots, x_{n}, \Lambda>1$ are real numbers, then the following inequalities hold

$$
\sum_{i=1}^{n}\left|x_{i}\right| \geq\left(\sum_{i=1}^{n}\left|x_{i}\right|^{2}\right)^{1 / 2}, \sum_{i=1}^{n}\left|x_{i}\right|^{\Lambda+1} \geq n^{(1-\Lambda) / 2}\left(\sum_{i=1}^{n}\left|x_{i}\right|^{2}\right)^{(\Lambda+1) / 2} .
$$

Proof. It is obvious that

$$
\left(\left|x_{1}\right|+\left|x_{2}\right|+\cdots+\left|x_{n}\right|\right)^{2} \geq x_{1}^{2}+x_{2}^{2}+\cdots+x_{n}^{2},
$$

which follows

$$
\sum_{i=1}^{n}\left|x_{i}\right| \geq\left(\sum_{i=1}^{n}\left|x_{i}\right|^{2}\right)^{1 / 2}
$$

For constants $p>1, q=\frac{p}{p-1}, \Upsilon_{i}>0(i=1,2, \ldots, n)$, it follows from the Höder inequality that

$$
\sum_{i=1}^{n} \Upsilon_{i}\left|x_{i}\right|=\sum_{i=1}^{n} \Upsilon_{i}^{1 / q} \Upsilon_{i}^{1 / p}\left|x_{i}\right| \leq\left(\sum_{i=1}^{n} \Upsilon_{i}\right)^{1 / q}\left(\sum_{i=1}^{n} \Upsilon_{i}\left|x_{i}\right|^{p}\right)^{1 / p}
$$

Choose $\Upsilon_{i}=1(i=1,2, \ldots, n)$, then we obtain

$$
\left(\sum_{i=1}^{n}\left|x_{i}\right|\right)^{p} \leq n^{p / q} \sum_{i=1}^{n}\left|x_{i}\right|^{p} .
$$

Further choose $p=(\Lambda+1) / 2>1, q=\frac{\Lambda+1}{\Lambda-1},(12)$ is equals to

$$
\left(\sum_{i=1}^{n}\left|x_{i}\right|\right)^{(\Lambda+1) / 2} \leq n^{(\Lambda-1) / 2} \sum_{i=1}^{n}\left|x_{i}\right|^{(\Lambda+1) / 2} .
$$

Then it is easy to get

$$
\sum_{i=1}^{n}\left|x_{i}\right|^{\Lambda+1} \geq n^{(1-\Lambda) / 2}\left(\sum_{i=1}^{n}\left|x_{i}\right|^{2}\right)^{(\Lambda+1) / 2} .
$$

The proof is completed.

Lemma 2. If function $F(t):[0,+\infty) \rightarrow R$ is absolute continuous and $\lim _{t \rightarrow+\infty} \int_{0}^{t} F(s) \mathrm{d} s$ exists and is bounded, then $F(t) \rightarrow 0$ as $t \rightarrow+\infty$.

Proof. Since function $F(t):[0,+\infty) \rightarrow R$ is absolute continuous, then it is also uniformly continuous, which means $\forall \epsilon>0, \exists \delta(\epsilon)>0, \forall x, t \geq 0,|x-t|<\delta$, such that

$$
|F(x)-F(t)|<\frac{\epsilon}{2} .
$$

Then we have

$$
\int_{x}^{x+\delta}\left(F(x)-\frac{\epsilon}{2}\right) \mathrm{d} t<\int_{x}^{x+\delta} F(t) \mathrm{d} t<\int_{x}^{x+\delta}\left(F(x)+\frac{\epsilon}{2}\right) \mathrm{d} t,
$$

and

$$
\frac{1}{\delta} \int_{x}^{x+\delta}|F(x)-F(t)| \mathrm{d} t<\frac{\epsilon}{2} .
$$

On the other hand, $\lim _{t \rightarrow+\infty} \int_{0}^{t} F(s) \mathrm{d} s$ exists and is bounded, then for big enough $x$ we have

$$
\int_{x}^{x+\delta}|F(t)| \mathrm{d} t<\frac{\delta \epsilon}{2}
$$

Thus from (17) and (18), for $\forall \epsilon>0, \exists \Pi>0, x>\Pi$, we can get

$$
\begin{aligned}
|F(x)| & =\frac{1}{\delta} \int_{x}^{x+\delta}|F(x)| \mathrm{d} t \\
& \leq \frac{1}{\delta} \int_{x}^{x+\delta}|F(x)-F(t)| \mathrm{d} t+\frac{1}{\delta} \int_{x}^{x+\delta}|F(t)| \mathrm{d} t
\end{aligned}
$$




$$
\leq \frac{\epsilon}{2}+\frac{\epsilon}{2}=\epsilon
$$

It is obvious that $\lim _{t \rightarrow+\infty} F(t)=0$. The proof is completed.

Remark 2. This lemma is the extension of Barbalat lemma. Together with the Lyapunov theory, it is successfully applied to applications of the adaptive control, parameter estimation and absolute stability. In this paper, it is adopted to prove the asymptotical stability of the error system.

\section{Main results}

In this section, we will address the controller design problem for fixed-time synchronization study of DNNs with discontinuous activations. Then we will show the designed two control methods including the adaptive and state control, and show how the two control methods take effect in the fixed-time synchronization study.

First an adaptive controller is designed as follows.

$$
\begin{aligned}
u_{i}(t)= & -\alpha_{i}(t) e_{i}(t)-\beta_{i}(t) \operatorname{sign}\left(e_{i}(t)\right) \\
& -\gamma_{i} \operatorname{sign}\left(e_{i}(t)\right)\left(\sum_{j=1}^{n}\left|e_{j}(t-\tau(t))\right|+\left|e_{i}(t)\right|^{\varepsilon}\right),
\end{aligned}
$$

and the adaptive updated law is

$$
\begin{aligned}
\dot{\alpha}_{i}(t)= & \delta_{i} e_{i}^{2}(t)-\eta_{i} \operatorname{sign}\left(\alpha_{i}(t)-\pi_{i}\right) \\
& -\theta_{i} \operatorname{sign}\left(\alpha_{i}(t)-\pi_{i}\right)\left|\alpha_{i}(t)-\pi_{i}\right|^{\varepsilon}, \\
\dot{\beta}_{i}(t)= & \vartheta_{i}\left|e_{i}(t)\right|-\sigma_{i} \operatorname{sign}\left(\beta_{i}(t)-\varpi_{i}\right) \\
& -v_{i} \operatorname{sign}\left(\beta_{i}(t)-\varpi_{i}\right)\left|\beta_{i}(t)-\varpi_{i}\right|^{\varepsilon},
\end{aligned}
$$

where $\gamma_{i}, \delta_{i}, \eta_{i}, \vartheta_{i}, \sigma_{i}, \pi_{i}, \varpi_{i}, \theta_{i}, v_{i}, \varepsilon$ are constants for $i=$ $1,2, \ldots, n$.

Under the controller (20), the error system (7) is transformed into:

$$
\begin{aligned}
\dot{e}_{i}(t)= & -d_{i} e_{i}(t)+\sum_{j=1}^{n} a_{i j} \zeta_{j}(t)+\sum_{j=1}^{n} b_{i j} \zeta_{j}(t-\tau(t)), \\
& -\alpha_{i}(t) e_{i}(t)-\beta_{i}(t) \mu_{i}(t) \\
& -\gamma_{i} \mu_{i}(t)\left(\sum_{j=1}^{n}\left|e_{j}(t-\tau(t))\right|+\left|e_{i}(t)\right|^{\varepsilon}\right), \\
& \text { for } \text { a.a. } t \geq 0, i=1,2, \ldots, n
\end{aligned}
$$

where $\mu_{i}(t)=\operatorname{sign}\left(e_{i}(t)\right)$, if $e_{i}(t) \neq 0$, while $\mu_{i}(t)$ can be arbitrarily chosen in $[-1,1]$, if $e_{i}(t)=0 . \alpha_{i}(t), \beta_{i}(t), \gamma_{i}$ are defined by the controller (20), and the other parameters are the same as in (7).

Theorem 1. Suppose that Assumptions (A1) and (A2) hold, DNNs (1) and (5) are globally synchronized in fixed time via the controller (20), if constants $\varepsilon$ and $\gamma_{i}$ in (20) satisfy $\varepsilon>1$,

$$
\max \left\{\left|b_{i j}\right| h_{j}-\gamma_{i}\right\} \leq 0,
$$

for all $i, j=1,2, \ldots, n$. Moreover, the settling time functional $T_{0}(\phi) \leq \frac{\sqrt{2}}{\rho}+\frac{2^{(1-\varepsilon) / 2}}{n^{(1-\varepsilon) / 2} \xi(\varepsilon-1)}$, where $\rho=\min \{\hat{\rho}, \check{\rho}, 1\}>$
$0, \hat{\rho}=\min \left\{\eta_{i} \delta_{i}^{-1 / 2}\right\}, \check{\rho}=\min \left\{\sigma_{i} \vartheta_{i}^{-1 / 2}\right\}, \xi=\min \{\hat{\xi}, \check{\xi}, \gamma\}>$

$0, \hat{\xi}=\min \left\{\theta_{i} \delta_{i}^{(\varepsilon+1) / 2}\right\}, \breve{\xi}=\min \left\{v_{i} \vartheta_{i}^{(\varepsilon+1) / 2}\right\}, \gamma=\min \left\{\gamma_{i}\right\}$ for all $i=1,2, \ldots, n$.

Proof. Consider

$$
\begin{aligned}
V(t)= & \frac{1}{2} \sum_{i=1}^{n}\left[e_{i}^{2}(t)+\frac{1}{\delta_{i}}\left(\alpha_{i}(t)-\pi_{i}\right)^{2}\right. \\
& \left.+\frac{1}{\vartheta_{i}}\left(\beta_{i}(t)-\varpi_{i}\right)^{2}\right],
\end{aligned}
$$

where $\delta_{i}>0, \vartheta_{i}>0, \pi_{i}, \varpi_{i}$ are constants, and $\delta_{i}, \vartheta_{i}, \pi_{i}, \varpi_{i}$ are defined by the controller (20).

By calculating the time derivative of function (23) along the trajectories of (21) according to the Chain Rule given in Efimov et al. (2014), function $V(t)$ is differentiable for a.a. $t \geq 0$ and for a.a. $t \in[0,+\infty)$

$$
\begin{aligned}
D^{+} V(t)= & \sum_{i=1}^{n}\left[e_{i}(t) \dot{e}_{i}(t)+\frac{1}{\delta_{i}}\left(\alpha_{i}(t)-\pi_{i}\right) \dot{\alpha}_{i}(t)\right. \\
& \left.+\frac{1}{\vartheta_{i}}\left(\beta_{i}(t)-\varpi_{i}\right) \dot{\beta}_{i}(t)\right] \\
= & \sum_{i=1}^{n}\left[-d_{i} e_{i}^{2}(t)+\sum_{j=1}^{n} a_{i j} \zeta_{j}(t) e_{i}(t)\right. \\
& +\sum_{j=1}^{n} b_{i j} \zeta_{j}(t-\tau(t)) e_{i}(t)-\alpha_{i}(t) e_{i}^{2}(t)-\beta_{i}(t) \mu_{i}(t) e_{i}(t) \\
& -\gamma_{i} \mu_{i}(t) e_{i}(t)\left(\sum_{j=1}^{n}\left|e_{j}(t-\tau(t))\right|+\left|e_{i}(t)\right|^{\varepsilon}\right) \\
& \left.+\frac{1}{\delta_{i}}\left(\alpha_{i}(t)-\pi_{i}\right) \dot{\alpha}_{i}(t)+\frac{1}{\vartheta_{i}}\left(\beta_{i}(t)-\varpi_{i}\right) \dot{\beta}_{i}(t)\right]
\end{aligned}
$$

Based on the assumptions (A1) and (A2), we get the following two inequalities

$$
\begin{aligned}
& \sum_{i=1}^{n} \sum_{j=1}^{n} a_{i j} \zeta_{j}(t) e_{i}(t) \\
\leq & \sum_{i=1}^{n} \sum_{j=1}^{n}\left|a_{i j}\right|\left|\zeta_{j}(t)\right|\left|e_{i}(t)\right| \\
\leq & \sum_{i=1}^{n} \sum_{j=1}^{n}\left|a_{i j}\right|\left|e_{i}(t)\right|\left(h_{j}\left|e_{j}(t)\right|+r_{j}\right) \\
\leq & \sum_{i=1}^{n} \sum_{j=1}^{n} h_{j}\left|a_{i j}\right|\left|e_{i}(t) \| e_{j}(t)\right|+\sum_{i=1}^{n} \sum_{j=1}^{n} r_{j}\left|a_{i j}\right|\left|e_{i}(t)\right| \\
\leq & \frac{1}{2} \sum_{i=1}^{n} \sum_{j=1}^{n}\left(\left|a_{i j}\right| h_{j}+\left|a_{j i}\right| h_{i}\right) e_{i}^{2}(t) \\
& +\sum_{i=1}^{n} \sum_{j=1}^{n}\left|a_{i j}\right| r_{j}\left|e_{i}(t)\right|, \\
& \sum_{i=1}^{n} \sum_{j=1}^{n} b_{i j} \zeta_{j}(t-\tau(t)) e_{i}(t) \\
\leq & \sum_{i=1}^{n} \sum_{j=1}^{n} h_{j}\left|b_{i j}\right|\left|e_{i}(t) \| e_{j}(t-\tau(t))\right|
\end{aligned}
$$




$$
+\sum_{i=1}^{n} \sum_{j=1}^{n}\left|b_{i j}\right| r_{j}\left|e_{i}(t)\right|
$$

By combining with (24)-(26), we derive

$$
\begin{aligned}
& D^{+} V(t) \leq \sum_{i=1}^{n}\left[-d_{i} e_{i}^{2}(t)+\frac{1}{2} \sum_{j=1}^{n}\left(\left|a_{i j}\right| h_{j}+\left|a_{j i}\right| h_{i}\right) e_{i}^{2}(t)\right. \\
& +\sum_{j=1}^{n} h_{j}\left|b_{i j}\left\|e_{i}(t)\right\| e_{j}(t-\tau(t))\right| \\
& +\sum_{j=1}^{n}\left(\left|a_{i j}\right|+\left|b_{i j}\right|\right) r_{j}\left|e_{i}(t)\right|-\alpha_{i}(t) e_{i}^{2}(t) \\
& -\beta_{i}(t)\left|e_{i}(t)\right|-\gamma_{i} \sum_{j=1}^{n}\left|e_{j}(t-\tau(t))\right|\left|e_{i}(t)\right|-\gamma_{i}\left|e_{i}(t)\right|^{\varepsilon+1} \\
& \left.+\frac{1}{\delta_{i}}\left(\alpha_{i}(t)-\pi_{i}\right) \dot{\alpha}_{i}(t)+\frac{1}{\vartheta_{i}}\left(\beta_{i}(t)-\varpi_{i}\right) \dot{\beta}_{i}(t)\right] \\
& \leq \sum_{i=1}^{n}\left[-d_{i} e_{i}^{2}(t)+\frac{1}{2} \sum_{j=1}^{n}\left(\left|a_{i j}\right| h_{j}+\left|a_{j i}\right| h_{i}\right) e_{i}^{2}(t)\right. \\
& +\sum_{j=1}^{n}\left(\left|b_{i j}\right| h_{j}-\gamma_{i}\right)\left|e_{i}(t)\right|\left|e_{j}(t-\tau(t))\right| \\
& +\sum_{j=1}^{n}\left(\left|a_{i j}\right|+\left|b_{i j}\right|\right) r_{j}\left|e_{i}(t)\right|-\gamma_{i}\left|e_{i}(t)\right|^{\varepsilon+1} \\
& -\pi_{i} e_{i}^{2}(t)-\frac{\eta_{i}}{\delta_{i}}\left|\alpha_{i}(t)-\pi_{i}\right|-\frac{\theta_{i}}{\delta_{i}}\left|\alpha_{i}(t)-\pi_{i}\right|^{\varepsilon+1} \\
& \left.-\varpi_{i}\left|e_{i}(t)\right|-\frac{\sigma_{i}}{\vartheta_{i}}\left|\beta_{i}(t)-\varpi_{i}\right|-\frac{v_{i}}{\vartheta_{i}}\left|\beta_{i}(t)-\varpi_{i}\right|^{\varepsilon+1}\right] \\
& \leq \sum_{i=1}^{n} e_{i}^{2}(t)\left[-\left(d_{i}+\pi_{i}\right)+\frac{1}{2} \sum_{j=1}^{n}\left(\left|a_{i j}\right| h_{j}+\left|a_{j i}\right| h_{i}\right)\right] \\
& +\sum_{i=1}^{n} \sum_{j=1}^{n}\left(\left|b_{i j}\right| h_{j}-\gamma_{i}\right)\left|e_{j}(t-\tau(t))\right|\left|e_{i}(t)\right| \\
& -\sum_{i=1}^{n}\left[\frac{\eta_{i}}{\delta_{i}}\left|\alpha_{i}(t)-\pi_{i}\right|+\frac{\sigma_{i}}{\vartheta_{i}}\left|\beta_{i}(t)-\varpi_{i}\right|\right. \\
& \left.+\left(\varpi_{i}-\sum_{j=1}^{n}\left(\left|a_{i j}\right|+\left|b_{i j}\right|\right) r_{j}\right)\left|e_{i}(t)\right|\right] \\
& -\sum_{i=1}^{n}\left[\gamma_{i}\left|e_{i}(t)\right|^{\varepsilon+1}+\frac{\theta_{i}}{\delta_{i}}\left|\alpha_{i}(t)-\pi_{i}\right|^{\varepsilon+1}\right. \\
& \left.+\frac{v_{i}}{\vartheta_{i}}\left|\beta_{i}(t)-\varpi_{i}\right|^{\varepsilon+1}\right] \text {. }
\end{aligned}
$$

Based on Lemma 1, we have the following inequalities

$$
\begin{aligned}
& {\left[\sum_{i=1}^{n}\left(\frac{1}{\delta_{i}}\left(\alpha_{i}(t)-\pi_{i}\right)^{2}+\frac{1}{\vartheta_{i}}\left(\beta_{i}(t)-\varpi_{i}\right)^{2}+e_{i}^{2}(t)\right)\right]^{1 / 2} } \\
\leq & \sum_{i=1}^{n}\left[\left|\alpha_{i}(t)-\pi_{i}\right| / \delta_{i}^{1 / 2}+\left|\beta_{i}(t)-\varpi_{i}\right| / \vartheta_{i}^{1 / 2}+\left|e_{i}(t)\right|\right] \\
& n^{(1-\varepsilon) / 2}\left[\sum_{i=1}^{n}\left(\frac{1}{\delta_{i}}\left(\alpha_{i}(t)-\pi_{i}\right)^{2}+\frac{1}{\vartheta_{i}}\left(\beta_{i}(t)-\varpi_{i}\right)^{2}+e_{i}^{2}(t)\right)\right]^{(\varepsilon+1) / 2}
\end{aligned}
$$

$\leq \sum_{i=1}^{n}\left[\left|\alpha_{i}(t)-\pi_{i}\right| / \delta_{i}^{(\varepsilon+1) / 2}+\left|\beta_{i}(t)-\varpi_{i}\right| / \vartheta_{i}^{(\varepsilon+1) / 2}+\left|e_{i}(t)\right|^{\varepsilon+1}\right]$

Substituting (28) and (29) into (27), and if the constants $\pi_{i}$ and $\varpi_{i}$ are properly chosen such that $\pi_{i}=1 / 2 \sum_{j=1}^{n}\left(\left|a_{i j}\right| h_{j}+\right.$ $\left.\left|a_{j i}\right| h_{i}\right)-d_{i}, \varpi_{i}=\sum_{j=1}^{n}\left(\left|a_{i j}\right|+\left|b_{i j}\right|\right) r_{j}+1$, then for $a . a . t \in[0,+\infty)$, we have

$$
\begin{aligned}
D^{+} V(t) \leq & -\sum_{i=1}^{n}\left[\frac{\eta_{i}}{\delta_{i}}\left|\alpha_{i}(t)-\pi_{i}\right|+\frac{\sigma_{i}}{\vartheta_{i}}\left|\beta_{i}(t)-\varpi_{i}\right|+\left|e_{i}(t)\right|\right] \\
& -\sum_{i=1}^{n}\left[\gamma_{i}\left|e_{i}(t)\right|^{\varepsilon+1}+\frac{\theta_{i}}{\delta_{i}}\left|\alpha_{i}(t)-\pi_{i}\right|^{\varepsilon+1}\right. \\
& \left.+\frac{v_{i}}{\vartheta_{i}}\left|\beta_{i}(t)-\varpi_{i}\right|^{\varepsilon+1}\right] \\
\leq & -\rho[2 V(t)]^{1 / 2}-(3 n)^{(1-\varepsilon) / 2} \xi[2 V(t)]^{(\varepsilon+1) / 2}
\end{aligned}
$$

where $\rho=\min \{\hat{\rho}, \check{\rho}, 1\}>0, \hat{\rho}=\min \left\{\eta_{i} \delta_{i}^{-1 / 2}\right\}, \check{\rho}=$ $\min \left\{\sigma_{i} \vartheta_{i}^{-1 / 2}\right\}, \xi=\min \{\hat{\xi}, \breve{\xi}, \gamma\}>0, \hat{\xi}=\min \left\{\theta_{i} \delta_{i}^{(\varepsilon-1) / 2}\right\}, \check{\xi}=$ $\min \left\{v_{i} \vartheta_{i}^{(\varepsilon-1) / 2}\right\}, \gamma=\min \left\{\gamma_{i}\right\}$ for all $i=1,2, \ldots, n$.

Since any solution $e(t)$ of system (21) is absolute continuous, function $V(t)$ is absolute continuous. From (30), we can get $D^{+} V(t) \leq-\rho[2 V(t)]^{1 / 2}$ for a.a. $t \in[0,+\infty)$ and $\int_{0}^{t} \rho[2 V(s)]^{1 / 2} \mathrm{~d} s \leq V(0)-V(t)$. Then function $\digamma(t)=$ $\int_{0}^{t} \rho[2 V(s)]^{1 / 2} \mathrm{~d} s$ is monotonic increasing and $\digamma(t) \leq V(0, \phi)$, which means $\lim _{t \rightarrow+\infty} \int_{0}^{t} \rho[2 V(s)]^{1 / 2} \mathrm{~d} s$ exists and is bounded. From lemma 2, we conclude that $V(t) \rightarrow 0$ as $t \rightarrow+\infty$. Together with (23), we can get the error $e(t) \rightarrow 0$ as $t \rightarrow+\infty$, which means zero solution of the error system is globally asymptotically stable.

From (30) and for a.a. $t \in[0,+\infty)$, we have $D^{+} V(t) \leq-\rho[2 V(t)]^{1 / 2}$ if $V(t) \leq 1$ and $D^{+} V(t) \leq$ $-(3 n)^{(1-\varepsilon) / 2} \xi[2 V(t)]^{(\varepsilon+1) / 2}$ if $V(t) \geq 1$. Hence for any $e(t)$ of system $(21)$ such that $V(0, \phi)>1$ or $V(t) \leq 1$, we can get $V(t)=0$ for all $t \geq T_{\text {max }}=\frac{\sqrt{2}}{\rho}+\frac{2^{(1-\varepsilon) / 2}}{(3 n)^{(1-\varepsilon) / 2} \xi(\varepsilon-1)}$. Together with (23), we can get the error $e_{i}(t)=0$ for all $t \geq T_{\max }$, which means zero solution of the error system is globally fixed-time stable. Then DNNs (1) and (5) are globally fixed-time synchronized via the controller (20). The settling time functional $T_{0}(\phi) \leq \frac{\sqrt{2}}{\rho}+\frac{2^{(1-\varepsilon) / 2}}{(3 n)^{(1-\varepsilon) / 2} \xi(\varepsilon-1)}$. The proof is completed.

Remark 3. Compared with exponential or finite-time synchronization, fixed-time synchronization has its obvious advantages. Different from the exponential synchronization, fixedtime synchronization performs fast convergence speed, which meets the necessary requirement in some practical applications. Different from the finite-time synchronization, the settling time for fixed-time synchronization is independently on initial conditions. So it shows superiority when the initial synchronization errors of the drive-response systems are hard to calculate.

Remark 4. Recently, finite-time synchronization of DNNs and memristive DNNs were studied via the state feedback control 
(Abdurahman et al., 2015; Hu et al., 2014; Huang et al., 2014; Jiang et al., 2015; Velmurugan et al., 2016; Wang et al., 2016; Yang et al., 2015). Also fixed-time synchronization of CohenGrossberg DNNs was investigated with delayed state control in Wan et al. (2016). Different from the state control methods in the previous literature, an adaptive control method is adopted in studying the fixed-time synchronization of DNNs in our paper. It is noted that the adaptive controller can adjust coupling strength automatically and it gets effective results in practical applications. In addition, the criteria deduced by the adaptive control method for fixed-time synchronization of DNNs are simple and easy to be checked.

In the following, we propose a state feedback control method and then we will show the different role in the fixed-time synchronization study of DNNs.

Another state feedback controller is designed as follows.

$$
\begin{aligned}
u_{i}(t)= & -\alpha_{i} e_{i}(t)-\beta_{i} \operatorname{sign}\left(e_{i}(t)\right) \\
& -\gamma_{i} \operatorname{sign}\left(e_{i}(t)\right)\left(\sum_{j=1}^{n}\left|e_{j}(t-\tau(t))\right|+\left|e_{i}(t)\right|^{\varepsilon}\right),
\end{aligned}
$$

where $\alpha_{i}, \beta_{i}, \gamma_{i}, \varepsilon$ are constants for $i=1,2, \ldots, n$.

Under the controller (31), the error system (7) is transformed into:

$$
\begin{aligned}
\dot{e}_{i}(t)= & -d_{i} e_{i}(t)+\sum_{j=1}^{n} a_{i j} \zeta_{j}(t)+\sum_{j=1}^{n} b_{i j} \zeta_{j}(t-\tau(t))-\alpha_{i} e_{i}(t) \\
& -\beta_{i} \mu_{i}(t)-\gamma_{i} \mu_{i}(t)\left(\sum_{j=1}^{n}\left|e_{j}(t-\tau(t))\right|+\left|e_{i}(t)\right|^{\varepsilon}\right),
\end{aligned}
$$

for a.a. $t \geq 0, i=1,2, \ldots, n$,

where $\alpha_{i}, \beta_{i}, \gamma_{i}$ are defined by the controller (31) and the other parameters are the same as in (21).

Remark 5. The discontinuous function $\operatorname{sign}\left(e_{i}(t)\right)$ in (20) and (31) will result in some undesirable oscillations known as the chattering. So in order to attenuate the problem in practical applications, two functions are used to replace the discontinuous function $\operatorname{sign}\left(e_{i}(t)\right)$. One is a saturation function $\operatorname{sat}\left(e_{i}(t)\right)$ which take value as $e_{i}(t)$ if $\left|e_{i}(t)\right| \leq 1$ and $\operatorname{sign}\left(e_{i}(t)\right)$ if $\left|e_{i}(t)\right|>1$. The other function is $e_{i}(t) /\left(\left|e_{i}(t)\right|+\epsilon_{i}\right)\left(\epsilon_{i}>0\right.$ is a sufficiently small constant).

Theorem 2. Suppose that Assumptions (A1) and (A2) hold, DNNs (1) and (5) are globally synchronized in fixed time via the controller (31), if constants $\varepsilon>1, \alpha_{i}, \beta_{i}$ and $\gamma_{i}$ in (31) satisfy

$$
\begin{aligned}
& d_{i}+\alpha_{i}-\frac{1}{2} \sum_{j=1}^{n}\left(\left|a_{i j}\right| h_{j}+\left|a_{j i}\right| h_{i}\right)>0, \\
& \beta_{i}-\sum_{j=1}^{n}\left(\left|a_{i j}\right|+\left|b_{i j}\right|\right) r_{j}>0 \\
& \max \left\{\left|b_{i j}\right| h_{j}-\gamma_{i}\right\} \leq 0
\end{aligned}
$$

for all $i, j=1,2, \ldots, n$. Moreover, the settling time functional $T_{0}(\phi) \leq \frac{\sqrt{2}}{\varrho}+\frac{2^{(1-\varepsilon) / 2}}{n^{(1-\varepsilon) / 2} \gamma(\varepsilon-1)}$, where $\varrho=\min \left\{\beta_{i}-\sum_{j=1}^{n}\left(\left|a_{i j}\right|+\right.\right.$ $\left.\left.\left|b_{i j}\right|\right) r_{j}\right\}>0, \gamma=\min \left\{\gamma_{i}\right\}$ for all $i=1,2, \ldots, n$.
Proof. Consider

$$
V(t)=\frac{1}{2} \sum_{i=1}^{n} e_{i}^{2}(t)
$$

By calculating the time derivative of function (36) along the trajectories of (32) according to the Chain Rule given in Efimov et al. (2014), function $V(t)$ is differentiable for a.a. $t \geq 0$ and for a.a. $t \in[0,+\infty)$

$$
\begin{aligned}
D^{+} V(t)= & \sum_{i=1}^{n} e_{i}(t) \dot{e}_{i}(t) \\
= & \sum_{i=1}^{n}\left[-d_{i} e_{i}^{2}(t)+\sum_{j=1}^{n} a_{i j} \zeta_{j}(t) e_{i}(t)\right. \\
& +\sum_{j=1}^{n} b_{i j} \zeta_{j}(t-\tau(t)) e_{i}(t)-\alpha_{i} e_{i}^{2}(t)-\beta_{i} \mu_{i}(t) e_{i}(t) \\
& \left.-\gamma_{i} \mu_{i}(t) e_{i}(t)\left(\sum_{j=1}^{n}\left|e_{j}(t-\tau(t))\right|+\left|e_{i}(t)\right|^{\varepsilon}\right)\right] .
\end{aligned}
$$

By combining with (25), (26) and (37), we derive

$$
\begin{aligned}
D^{+} V(t) \leq & \sum_{i=1}^{n}\left[-d_{i} e_{i}^{2}(t)+\frac{1}{2} \sum_{j=1}^{n}\left(\left|a_{i j}\right| h_{j}+\left|a_{j i}\right| h_{i}\right) e_{i}^{2}(t)\right] \\
& +\left[\sum_{j=1}^{n} h_{j}\left|b_{i j}\right|\left|e_{i}(t)\right|\left|e_{j}(t-\tau(t))\right|\right. \\
& +\sum_{j=1}^{n}\left(\left|a_{i j}\right|+\left|b_{i j}\right|\right) r_{j}\left|e_{i}(t)\right|-\alpha_{i} e_{i}^{2}(t)-\beta_{i}\left|e_{i}(t)\right| \\
& \left.-\gamma_{i} \sum_{j=1}^{n}\left|e_{j}(t-\tau(t))\right|\left|e_{i}(t)\right|-\gamma_{i}\left|e_{i}(t)\right|^{\varepsilon+1}\right] \\
\leq & \sum_{i=1}^{n} e_{i}^{2}(t)\left[-\left(d_{i}+\alpha_{i}\right)+\frac{1}{2} \sum_{j=1}^{n}\left(\left|a_{i j}\right| h_{j}+\left|a_{j i}\right| h_{i}\right)\right] \\
& +\sum_{i=1}^{n} \sum_{j=1}^{n}\left(\left|b_{i j}\right| h_{j}-\gamma_{i}\right)\left|e_{j}(t-\tau(t))\right|\left|e_{i}(t)\right| \\
& -\sum_{i=1}^{n}\left(\beta_{i}-\sum_{j=1}^{n}\left(\left|a_{i j}\right|+\left|b_{i j}\right|\right) r_{j}\right)\left|e_{i}(t)\right| \\
& -\sum_{i=1}^{n} \gamma_{i}\left|e_{i}(t)\right|^{\varepsilon+1} .
\end{aligned}
$$

Then for a.a. $t \in[0,+\infty)$, we have

$$
D^{+} V(t) \leq-\varrho[2 V(t)]^{1 / 2}-\gamma n^{(1-\varepsilon) / 2}[2 V(t)]^{(\varepsilon+1) / 2},
$$

where $\varrho=\min \left\{\beta_{i}-\sum_{j=1}^{n}\left(\left|a_{i j}\right|+\left|b_{i j}\right|\right) r_{j}\right\}>0, \gamma=\min \left\{\gamma_{i}\right\}$ for all $i=1,2, \ldots, n$.

Similar to the proof of Theorem 1, the global fixed-time stability of (32) can be ensured, i.e. DNNs (1) and (5) are globally fixed-time synchronized via the controller (31). The settling time functional $T_{0}(\phi) \leq \frac{\sqrt{2}}{\varrho}+\frac{2^{(1-\varepsilon) / 2}}{n^{(1-\varepsilon) / 2} \gamma(\varepsilon-1)}$. The proof is completed. 
Remark 6. From Theorems 1 and 2, we can see that the two control methods are effective in realizing the fixed-time synchronization of DNNs. However, the results deduced by the two control methods are different. The criteria in Theorem 1 are more general and simple compared with those in Theorem 2. It also shows the superiority of the adaptive control method.

Remark 7. Finite-time convergence problem of neural networks was widely studied, see Abdurahman et al. (2015); Hu et al. (2014); Huang et al. (2014); Jiang et al. (2015); Liu et al. (2014); Mei et al. (2013); Shen \& Cao (2012); Velmurugan et al. (2016). Also Wan et al. (2016) discussed the fixed-time convergence of Cohen-Grossberg DNNs. It should be pointed out there are some restrictions in these works due to the fact that systems in Jiang et al. (2015); Liu et al. (2014); Shen \& Cao (2012) are without time delays and systems in Abdurahman et al. (2015); Hu et al. (2014); Huang et al. (2014); Mei et al. (2013); Velmurugan et al. (2016); Wan et al. (2016) are with continuous activations. Since our system contains the time delay and discontinuous activations, the results in this study are general compared with the previous work in Hu et al. (2014); Huang et al. (2014); Jiang et al. (2015); Liu et al. (2014); Mei et al. (2013); Shen \& Cao (2012); Velmurugan et al. (2016); Wan et al. (2016).

From the above results, we can see the parameter $\varepsilon$ plays important role in realizing fixed-time synchronization of DNNs. It is obvious that Theorems 1 and 2 are obtained by choosing $\varepsilon>1$ in the two controllers. By choosing $0 \leq \varepsilon<1$ in (20) and (31), we propose the following controllers with which the finite-time synchronization results can be derived directly from Theorems 1 and 2.

$$
\begin{aligned}
u_{i}(t)= & -\alpha_{i}(t) e_{i}(t)-\beta_{i}(t) \operatorname{sign}\left(e_{i}(t)\right) \\
& -\gamma_{i} \operatorname{sign}\left(e_{i}(t)\right)\left(\sum_{j=1}^{n}\left|e_{j}(t-\tau(t))\right|+\left|e_{i}(t)\right|^{\varepsilon}\right),
\end{aligned}
$$

and the adaptive updated law is

$$
\begin{aligned}
& \dot{\alpha}_{i}(t)=\delta_{i} e_{i}^{2}(t)-\eta_{i} \operatorname{sign}\left(\alpha_{i}(t)-\pi_{i}\right), \\
& \dot{\beta}_{i}(t)=\vartheta_{i}\left|e_{i}(t)\right|-\sigma_{i} \operatorname{sign}\left(\beta_{i}(t)-\varpi_{i}\right),
\end{aligned}
$$

where $\gamma_{i}, \delta_{i}, \eta_{i}, \vartheta_{i}, \sigma_{i}, \pi_{i}, \varpi_{i}, 0 \leq \varepsilon<1$ are constants for $i=$ $1,2, \ldots, n$.

Another state feedback controller is designed as follows.

$$
\begin{aligned}
u_{i}(t)= & -\alpha_{i} e_{i}(t)-\beta_{i} \operatorname{sign}\left(e_{i}(t)\right) \\
& -\gamma_{i} \operatorname{sign}\left(e_{i}(t)\right)\left(\sum_{j=1}^{n}\left|e_{j}(t-\tau(t))\right|+\left|e_{i}(t)\right|^{\varepsilon}\right),
\end{aligned}
$$

where $\alpha_{i}, \beta_{i}, \gamma_{i}, 0 \leq \varepsilon<1$ are constants for $i=1,2, \ldots, n$.

Theorem 3. Suppose that Assumptions (A1) and (A2) hold, DNNs (1) and (5) are globally synchronized in finite time via the controller (40) if constant $\gamma_{i}$ in (40) satisfy the condition (22) for all $i, j=1,2, \ldots, n$.

Theorem 4. Suppose that Assumptions (A1) and (A2) hold, DNNs (1) and (5) are globally synchronized in finite time via the controller (41) if conditions (33)-(35) hold for all $i, j=$ $1,2, \ldots, n$.

Remark 8. By choosing different values of the parameter $\varepsilon$ in the two controllers (20) and (31), Theorems 3 and 4 provide the finite-time convergence results for DNNs with discontinuous activations. Since the settling time functional can be calculated as the same in Wang et al. (2016), they are omitted in this paper. It is worth noting that if we choose $\varepsilon=0$ in the controller (41), then it turns into a general delayed controller which are used in Abdurahman et al. (2015); Hu et al. (2014); Wang et al. (2016).

\section{Numerical examples}

In this section, two examples are provided to verify the effectiveness of the results obtained in the previous section.

Example 1. Consider the two-dimensional DNNs

$$
\dot{x}(t)=-D x(t)+A f(x(t))+B f(x(t-\tau(t)))+I,
$$

where $x(t)=\left(x_{1}(t), x_{2}(t)\right)^{T}$ is the state vector, $D=$ $\operatorname{diag}(1,1), \tau(t)=e^{t} /\left(1+e^{t}\right), I=(0,0)^{T}$,

$$
\begin{aligned}
& A=\left[\begin{array}{cc}
3 & 5 \\
0.1 & 2
\end{array}\right], B=\left[\begin{array}{cc}
-2.5 & 0.2 \\
0.1 & -1.5
\end{array}\right] . \\
& f(x)= \begin{cases}\tanh (x)+0.02 x+0.028, & x>0, \\
\tanh (x)+0.02 x-0.028, & x<0 .\end{cases}
\end{aligned}
$$

Then from Assumption (A2) that $h_{j}=1.02, r_{j}=0.056$ for $j=1,2$. Under the initial condition $x_{1}(s)=0.8, x_{2}(s)=$ $-1.2, \forall s \in[-1,0)$, the phase plot of DNNs (42) is given in Fig.1. It is clear that DNNs (42) is a chaotic system which possesses complicated dynamic behaviors.

Consider DNNs (42) as the drive system, then the corresponding response system is

$$
\dot{z}(t)=-D z(t)+A f(z(t))+B f(z(t-\tau(t)))+I+u(t),
$$

where $z(t)=\left(z_{1}(t), z_{2}(t)\right)^{T}$ and the other parameters are the same as in DNNs (42), and the controller $u(t)=\left(u_{1}(t), u_{2}(t)\right)^{T}$ is determined later. Define the synchronization error $e_{i}(t)=$ $z_{i}(t)-x_{i}(t), i=1,2$. Under the initial condition $x_{1}(s)=$ $0.8, x_{2}(s)=-1.2, z_{1}(s)=2.8, z_{2}(s)=-2.8, \forall s \in[-1,0)$, Fig. 2 shows the state trajectories of errors $e_{1}(t)$ and $e_{2}(t)$ between DNNs (42) and (43) with $u(t)=(0,0)^{T}$. It is clear that the error system is unstable.

Now we choose $\gamma_{1}=\gamma_{2}=3, \varepsilon=1.5$ and $\delta_{i}=\eta_{i}=\theta_{i}=\vartheta_{i}=$ $\sigma_{i}=v_{i}=1, i=1,2$. Then the controller (20) is as follows

$$
\begin{aligned}
u_{i}(t)= & -\alpha_{i}(t) e_{i}(t)-\beta_{i}(t) \operatorname{sign}\left(e_{i}(t)\right) \\
& -3 \operatorname{sign}\left(e_{i}(t)\right)\left(\sum_{j=1}^{n}\left|e_{j}(t-\tau(t))\right|+\left|e_{i}(t)\right|^{1.5}\right), \\
& i=1,2,
\end{aligned}
$$

and the adaptive updated law is

$$
\dot{\alpha}_{i}(t)=e_{i}^{2}(t)-\operatorname{sign}\left(\alpha_{i}(t)-\pi_{i}\right)-\operatorname{sign}\left(\alpha_{i}(t)-\pi_{i}\right)\left|\alpha_{i}(t)-\pi_{i}\right|^{1.5},
$$




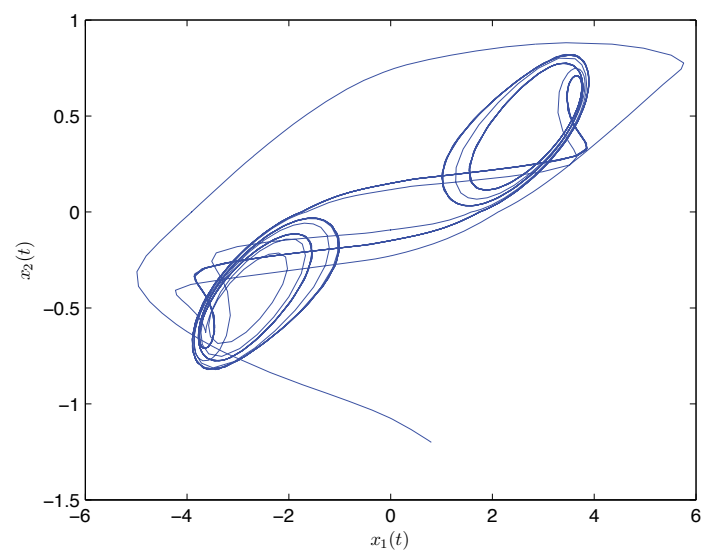

Figure 1: The phase plot of DNNs (42) with the initial condition $x_{1}(s)=$ $0.8, x_{2}(s)=-1.2, \forall s \in[-1,0)$.

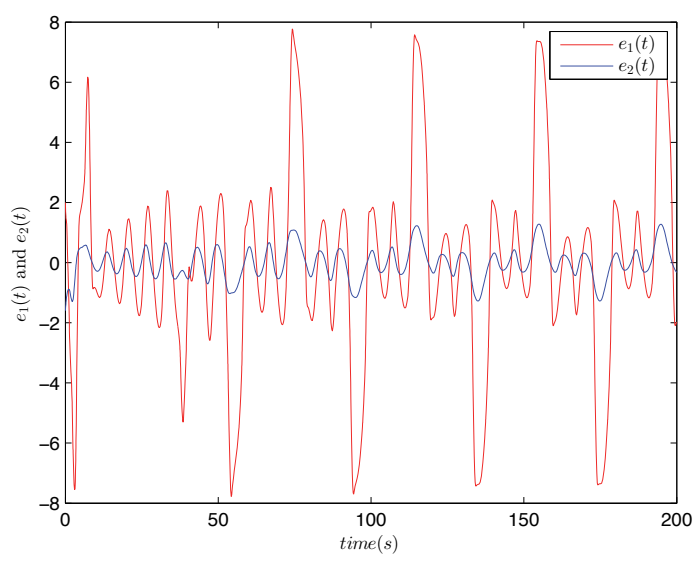

Figure 2: The state trajectories of errors $e_{1}(t)$ and $e_{2}(t)$ between DNNs (42) and (43) under the initial condition $x_{1}(s)=0.8, x_{2}(s)=-1.2, z_{1}(s)=2.8, z_{2}(s)=$ $-2.8, \forall s \in[-1,0)$ without any controllers.

$\dot{\beta}_{i}(t)=\left|e_{i}(t)\right|-\operatorname{sign}\left(\beta_{i}(t)-\varpi_{i}\right)-\operatorname{sign}\left(\beta_{i}(t)-\varpi_{i}\right)\left|\beta_{i}(t)-\varpi_{i}\right|^{1.5}$,

where $\pi_{1}=4.6610, \pi_{2}=3.6410, \varpi_{1}=2.5992, \varpi_{1}=2.2072$.

It is easy to check that the condition of Theorem 1 holds. Then it follows from Theorem 1 that DNNs (42) and (43) can be fixed-time synchronized via the controller (44), and $T_{\max }=4.0464(s)$. Under the controller (44), we can get the state trajectories of errors $e_{1}(t)$ and $e_{2}(t)$ which are illustrated by Fig.3. The states of the error system converge to zero in fixed-time, which shows the effectiveness of our results. The dynamical coupling strengthes $\alpha_{i}(t)$ and $\beta_{i}(t), i=1,2$ of the controller (44) are shown in Fig.4.

Remark 9. Different from the state control methods used in studying finite-time synchronization of DNNs (Abdurahman et al., 2015; Hu et al., 2014; Huang et al., 2014; Jiang et al., 2015; Velmurugan et al., 2016; Wang et al., 2016; Yang et al., 2015) or fixed-time synchronization of Cohen-Grossberg DNNs (Velmurugan et al., 2016), the adaptive control method is effective and the criteria it derived are simple and easy to verify. From (44), we can see that the parameters to be determined are only

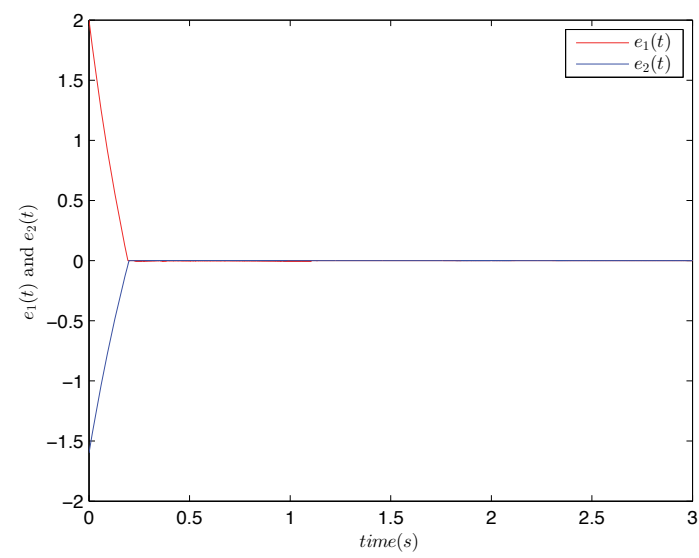

Figure 3: The state trajectories of errors $e_{1}(t)$ and $e_{2}(t)$ between DNNs (42) and (43) under the initial condition $\phi(s)=(2,-1.6)^{T}, \forall s \in[-1,0)$ with the controller (44).

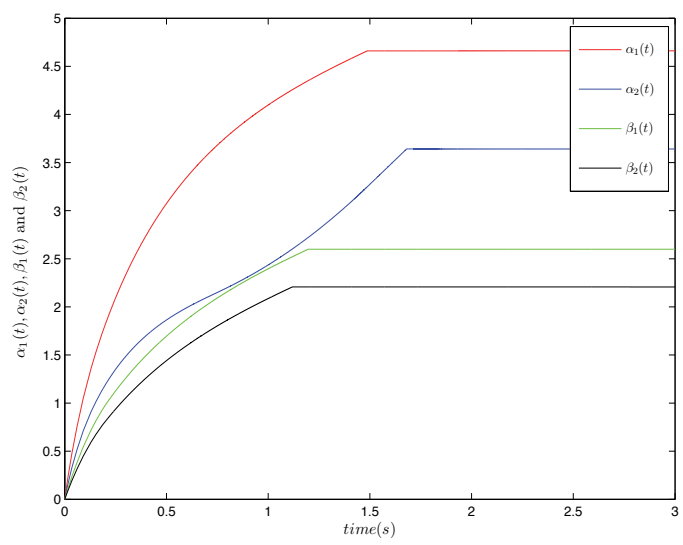

Figure 4: The dynamical coupling strengthes of the controller (44).

$\gamma_{1}$ and $\gamma_{2}$, which shows the superiority of the condition check of Theorem 1 .

Example 2. Consider another two-dimensional DNNs

$$
\dot{x}(t)=-D x(t)+A f(x(t))+B f(x(t-\tau(t)))+I,
$$

where $x(t)=\left(x_{1}(t), x_{2}(t)\right)^{T}$ is the state vector, $D=$ $\operatorname{diag}(1,1), \tau(t)=e^{t} /\left(1+e^{t}\right), I=(0,0)^{T}$,

$$
\begin{aligned}
& A=\left[\begin{array}{cc}
2 & -0.1 \\
-5 & 4.5
\end{array}\right], B=\left[\begin{array}{cc}
-1.5 & -0.1 \\
-0.2 & -4
\end{array}\right] . \\
& f(x)= \begin{cases}\tanh (x)+0.03, & x>0, \\
\tanh (x)-0.03, & x<0 .\end{cases}
\end{aligned}
$$

Then from Assumption (A2) that $h_{j}=1, r_{j}=0.06$ for $j=1,2$. Under the initial condition $x_{1}(s)=2.8, x_{2}(s)=-3, \forall s \in$ $[-1,0)$, the phase plot of DNNs (45) is given in Fig.5. It is clear that DNNs (45) is a chaotic system which possesses complicated dynamic behaviors.

Consider DNNs (45) as the drive system, then the corre- 


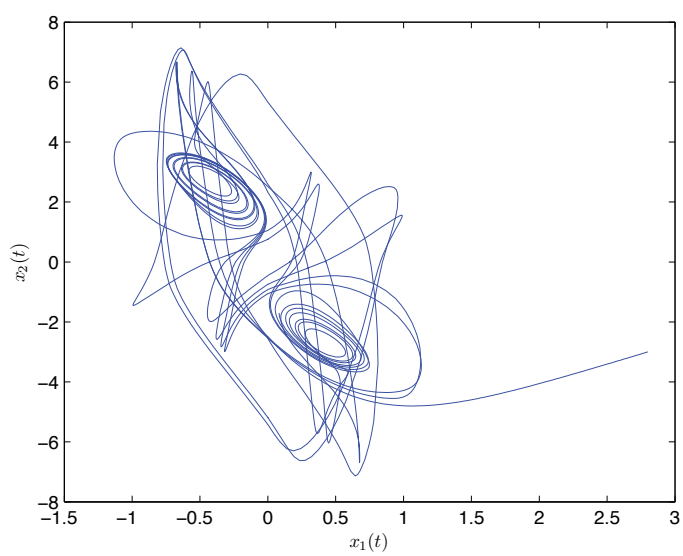

Figure 5: The phase plot of DNNs (45) with the initial condition $x_{1}(s)=$ $2.8, x_{2}(s)=-3, \forall s \in[-1,0)$.

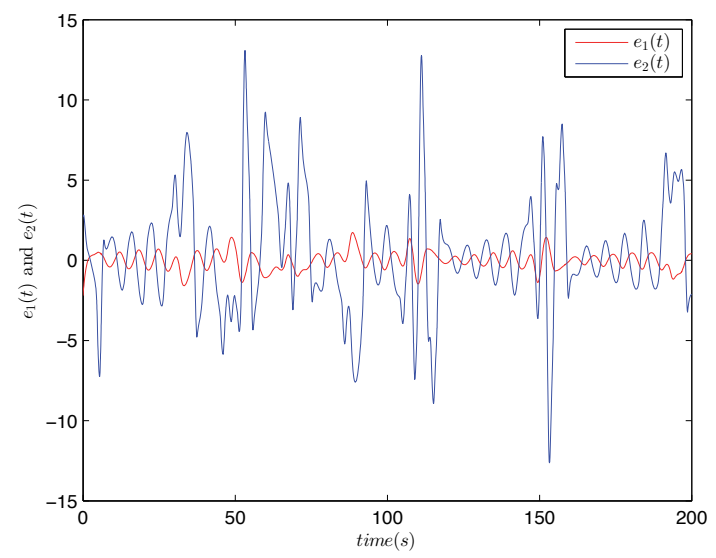

Figure 6: The state trajectories of errors $e_{1}(t)$ and $e_{2}(t)$ between DNNs (45) and (46) under the initial condition $x_{1}(s)=2.8, x_{2}(s)=-3, z_{1}(s)=0.6, z_{2}(s)=$ $-0.3, \forall s \in[-1,0)$ without any controllers.

sponding response system is

$$
\dot{z}(t)=-D z(t)+A f(z(t))+B f(z(t-\tau(t)))+I+u(t),
$$

where $z(t)=\left(z_{1}(t), z_{2}(t)\right)^{T}$ and the other parameters are the same as in (43), and the controller $u(t)=\left(u_{1}(t), u_{2}(t)\right)^{T}$ is determined later. Define the synchronization error $e_{i}(t)=z_{i}(t)-$ $x_{i}(t), i=1,2$. Under the initial condition $x_{1}(s)=2.8, x_{2}(s)=$ $-3, z_{1}(s)=0.6, z_{2}(s)=-0.3, \forall s \in[-1,0)$, Fig.6 shows the state trajectories of errors $e_{1}(t)$ and $e_{2}(t)$ between DNNs (45) and (46) with $u(t)=(0,0)^{T}$. It is clear that the error system is unstable.

Now we choose $\alpha_{1}=4, \alpha_{2}=6.5, \beta_{1}=1, \beta_{2}=1.5, \gamma_{1}=\gamma_{2}=$ $4, \varepsilon=1.5$. The controller (31) is as follows

$$
\left\{\begin{aligned}
u_{1}(t)=-4 e_{1}(t)-\operatorname{sign}\left(e_{1}(t)\right) & \\
& -4 \operatorname{sign}\left(e_{i}(t)\right)\left(\sum_{j=1}^{2}\left|e_{j}(t-\tau(t))\right|+\left|e_{i}(t)\right|^{1.5}\right), \\
u_{2}(t)= & -6.5 e_{2}(t)-1.5 \operatorname{sign}\left(e_{2}(t)\right) \\
& -4 \operatorname{sign}\left(e_{i}(t)\right)\left(\sum_{j=1}^{2}\left|e_{j}(t-\tau(t))\right|+\left|e_{i}(t)\right|^{1.5}\right) .
\end{aligned}\right.
$$

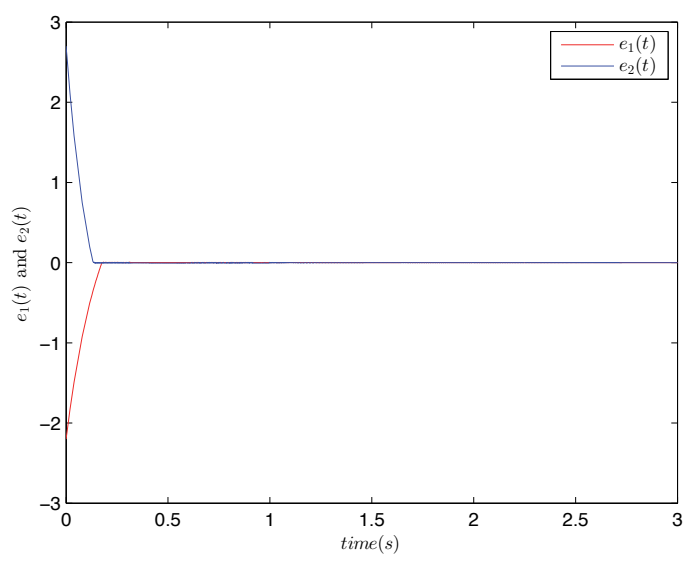

Figure 7: The state trajectories of errors $e_{1}(t)$ and $e_{2}(t)$ between DNNs (45) and (46) under the initial condition $\phi(s)=(-2.2,2.7)^{T}, \forall s \in[-1,0)$ with the controller (47).

Then we can get

$$
\begin{aligned}
& d_{1}+\alpha_{1}-\frac{1}{2} \sum_{j=1}^{2}\left(\left|a_{1 j}\right| h_{j}+\left|a_{j 1}\right| h_{1}\right)=0.45>0 \\
& d_{2}+\alpha_{2}-\frac{1}{2} \sum_{j=1}^{2}\left(\left|a_{2 j}\right| h_{j}+\left|a_{j 2}\right| h_{2}\right)=0.45>0 \\
& \beta_{1}-\sum_{j=1}^{2}\left(\left|a_{1 j}\right|+\left|b_{1 j}\right|\right) r_{j}=0.778>0 \\
& \beta_{2}-\sum_{j=1}^{2}\left(\left|a_{2 j}\right|+\left|b_{2 j}\right|\right) r_{j}=0.678>0
\end{aligned}
$$

It is easy to check that the conditions of Theorem 2 hold. Then it follows from Theorem 2 that DNNs (45) and (46) can be fixed-time synchronized via the controller (47), and $T_{\max }=$ $2.5859(s)$. Under the controller (47), we can get the state trajectories of errors $e_{1}(t)$ and $e_{2}(t)$ which are illustrated by Fig.7. The states of the error system converge to zero in fixed-time, which shows the effectiveness of our results.

\section{Conclusions}

The settling time functional for finite-time synchronization is heavily dependent on initial conditions, which means the time to converge to the equilibrium state is affected by the initial conditions. To overcome this problem, the fixed-time synchronization which is independently on initial conditions comes out. In this paper, the controller design problem for global fixedtime synchronization problem of DNNs with discontinuous activations has been discussed. To achieve the fixed-time synchronization, adaptive control and state feedback control laws have been adopted. Also some criteria have been established via the designed two control methods. We have shown that the two control methods are useful in dealing with the fixed-time synchronization of DNNs with discontinuous activations and 
are available for fixed-time or finite-time synchronization problem of other nonlinear time-delay systems. So the extension of obtained results for DNNs to other existing networks, such as complex networks and genetic regulatory networks will be our future study.

\section{Acknowledgments}

This work was supported by the Key Program of National Natural Science Foundation of China under Grant 61134012, the National Science Foundation of China under Grants 61673188, 61374085, and 61374150, and the Doctoral Program of Higher Education of China under Grant 20130142130012.

\section{References}

Abdurahman, A., Jiang, H., \& Teng, Z. (2015). Finite-time synchronization for memristor-based neural networks with time-varying delays, Neural Networks, 69, 20-28.

Bhat, S. P., \& Bernstein, D. S. (2000). Finite-time stability of continuous autonomous systems, SIAM Journal on Control and Optimization, 38, 751766.

Chandrasekar, A., Rakkiyappan, R., Rihan, F. A., \& Lakshmanan, S. (2014). Exponential synchronization of Markovian jumping neural networks with partly unknown transition probabilities via stochastic sampled-data control, Neurocomputing, 133, 385-398.

Chandrasekar, A., Rakkiyappan, R., \& Cao, J. (2015). Impulsive synchronization of Markovian jumping randomly coupled neural networks with partly unknown transition probabilities via multiple integral approach, Neural Networks, 70, 27-38.

Chen, X., \& Song, Q. (2010). Global exponential stability of the periodic solution of delayed Cohen-Grossberg neural networks with discontinuous activations, Neurocomputing, 73, 3097-3104.

Efimov, D., Polyakov, A., Fridman, E., Perruquetti, E., \& Richard, J. P. (2014). Comments on finite-time stability of time-delay systems, Automatica, 50, 1944-1947.

Forti, M., \& Nistri, P. (2003). Global convergence of neural networks with discontinuous neuron activations, IEEE Transactions on Circuits and Systems I: Fundamental Theory and Applications, 50, 1421-1435.

Forti, M., Nistri, P., \& Papini, D. (2005). Global exponential stability and global convergence in finite time of delayed neural networks with infinite gain, IEEE Transactions on Neural Networks and Learning Systems, 16, 14491463

Guo, Z., \& Huang, L. (2009). LMI conditions for global robust stability of delayed neural networks with discontinuous neuron activations, Applied Mathematics and Computation, 215, 889-900.

Hardy, G., Littlewood, J., \& Polya, G. (1988). Inequalities, Cambridge University Press: Cambridge.

Hu, C., Yu, J., \& Jiang, H. (2014). Finite-time synchronization of delayed neural networks with Cohen-Grossberg type based on delayed feedback control, Neurocomputing, 143, 90-96.

Huang, J., Li, C., Huang, T., \& He, X. (2014). Finite-time lag synchronization of delayed neural networks, Neurocomputing, 139, 145-149.

Jiang, M., Wang, S., Mei, J., \& Shen, Y. (2015). Finite-time synchronization control of a class of memristor-based recurrent neural networks, Neural Networks, 63, 133-140.

Karimi, H. R., \& Gao, H. (2010). New delay-dependent exponential synchronization for uncertain neural networks with mixed time delays, IEEE Transactions on Systems, Man, and Cybernetics, Part B: Cybernetics, 40, 173185.

Liu, X., Chen, T., Cao, J., \& Lu, W. (2011). Dissipativity and quasisynchronization for neural networks with discontinuous activations and parameter mismatches, Neural Networks, 24, 1013-1021.

Liu, X., Park, J. H., Jiang, N., \& Cao, J. (2014). Nonsmooth finite-time stabilization of neural networks with discontinuous activations, Nueral Networks, $52,25-32$.
Mei, J., Jiang, M., Wang, B., \& Long, B. (2013). Finite-time parameter identification and adaptive synchronization between two chaotic neural networks, Journal of the Franklin Institute, 350, 1617-1633.

Moulay, E., Dambrine, M., Yeganefar, N., \& Perruquetti, W. (2008). Finitetime stability and stabilization of time-delay systems, Systems and Control Letters, 57, 561-566.

Polyakov, A. (2012). Nonlinear feedback design for fixed-time stabilization of linear control systems, IEEE Transactions on Automatic Control, 57, 2106 2110.

Polyakov, A., Efimov, D., \& Perruquetti, W. (2015). Finite-time and fixed-time stabilization: Implicit Lyapunov function approach, Automatica, 51, 332 340 .

Shen, J., \& Cao, J. (2011). Finite-time synchronization of coupled neural networks via discontinuous controllers, Cognitive Neurodynamics, 5, 373-385.

Velmurugan, G., Rakkiyappan, R., \& Cao, J. (2016). Finite-time synchronization of fractional-order memristor-based neural networks with time delays, Neural Networks, 73, 36-46.

Wan, Y., Cao, J., Wen, G., \& Yu, W. (2016). Robust fixed-time synchronization of delayed Cohen-Grossberg neural networks, Neural Networks, 73, 86-94.

Wang, J., Huang, L., \& Guo, Z. (2009). Global asymptotic stability of neural networks with discontinuous activations, Neural Networks, 22, 931-937.

Wang, L., Shen, Y., \& Sheng, Y. (2016). Finite-time robust stabilization of uncertain delayed neural networks with discontinuous activations via delayed feedback control, Nueral Networks, 76, 46-54

Wang, L., Shen, Y., Yin, Q., \& Zhang, G. (2015). Adaptive synchronization of memristor-based neural networks with time-varying delays, IEEE Transactions on Neural Networks and Learning Systems, 26, 2033-2042.

Wang L., \& Shen, Y. (2015). Finite-time stabilizability and instabilizability of delayed memristive neural networks with nonlinear discontinuous controller, IEEE Transactions on Neural Networks and Learning Systems, 26, 2914-2924.

Wang, L., \& Shen, Y. (2015). Design of controller on synchronization of memristor-based neural networks with time-varying delays, Neurocomputing, 147, 372-379.

Wang, T., Xue, M., Fei, S., \& Li, T. (2013). Triple Lyapunov functional technique on delay-dependent stability for discrete-time dynamical networks, Neurocomputing, 122, 221-228.

Wen, S., Bao, G., Zeng, Z., Chen, Y., \& Huang, T. (2013). Global exponential synchronization of memristor-based recurrent neural networks with timevarying delays, Neural Networks, 48, 195-203.

Wu, A., Wen, S., \& Zeng, Z. (2012). Synchronization control of a class of memristor-based recurrent neural networks, Information Sciences, 183, 106116.

Wu, Z. G., Shi, P., Su, H., \& Chu, J. (2012). Exponential synchronization of neural networks with discrete and distributed delays under time-varying sampling, IEEE Transactions on Neural Networks and Learning Systems, 23, 1368-1376.

Yang, X., Song, Q., Liang, J., \& He, B. (2015). Finite-time synchronization of coupled discontinuous neural networks with mixed delays and nonidentical perturbations, Journal of the Franklin Institute, 352, 4382-4406.

Zhang, G., Shen, Y., \& Wang, L. (2013). Global anti-synchronization of a class of chaotic memristive neural networks with time-varying delays, Neural Networks, 46, 1-8.

Zhang, H., Xie, Y., Wang, Z., \& Zheng, C. (2007). Adaptive synchronization between two different chaotic neural networks with time delay, IEEE Transactions on Neural Networks, 18, 1841-1845.

Zhang, W., Li, C., Huang, T., \& Xiao, M. (2015). Synchronization of neural networks with stochastic perturbation via aperiodically intermittent control, Neural Networks, 71, 105-111. 